\title{
Pinch-off in the presence of surface-active polymers
}

\author{
M. RochÉ ${ }^{1}$ and H. KellaY ${ }^{2}$ \\ ${ }^{1}$ Department of Mechanical and Aerospace Engineering, Princeton University - Princeton, NJ 08544, USA \\ ${ }^{2}$ Université Bordeaux 1, Laboratoire Ondes et Matière d'Aquitaine, UMR 5798 CNRS - 351 cours de la Libération, \\ 33405 Talence, France, EU
}

PACS 47.20.Dr - Surface-tension-driven instability

PACS 47.20.Gv - Viscous and viscoelastic instabilities

PACS $47.57 . \mathrm{Ng}$ - Polymers and polymer solutions

\begin{abstract}
The rupture of fluid necks in a surrounding liquid in the presence of surface-active polymers shows a variety of changes with respect to the pure-fluid case. The role of the surface rheology due solely to the presence of such agents at the surface is uncovered by studying how the fluid neck radius approaches zero at pinch-off. The thinning dynamics of the neck turn out to be exponential in time. The thinning rate extracted from this dynamics is related to the surface viscosity of the polymer layer coating the interface and estimates of this quantity are presented.
\end{abstract}

(c)

The pinch-off of fluid necks during the detachment of a drop from an orifice is a classical nonlinear problem which combines hydrodynamics and capillarity. For Newtonian fluids, the thinning dynamics, which are characterized by measuring the evolution of the minimum radius of the neck $h_{\min } v s$. time $t$, follow temporal scaling laws which result from a subtle interplay between the capillary, viscous and inertial stresses. The shape of the neck shows self-similarity close to break-up [1,2]. Many droplet-based applications such as inkjet printing or the formation of emulsions require a good understanding of the pinch-off process. These applications often involve surface-active chemicals such as surfactants or polymers, e.g., to control the nature and the size of the emulsion and to stabilize the droplets against coalescence [3]. These molecules change the capillary, viscous and elastic properties of fluid-fluid interfaces, and should therefore influence the thinning of the neck.

While the effects of surfactant molecules on pinch-off dynamics have been studied theoretically [4], numerically [5-7] and experimentally [8], the impact of surfaceactive polymers has received no attention to the best of our knowledge. It is known, for example, that polymer laden interfaces may present important surface stresses [9]. Such stresses should lead to drastic effects on liquid bridge formation and rupture during pinch-off. In this letter, we present experimental evidence that the presence of surface-active polymers at a fluid-fluid interface modifies the thinning dynamics in a non-intuitive way. In particular, we observe the growth of beads-on-a-string structures, an exponential slowing-down of the thinning dynamics as rupture is approached and the inhibition of break-up. These observations, similar to those reported for bulk polymeric solutions [10-13], are surprising since the polymers lie only at the fluid-fluid interface in the case considered here. We suggest that these observations can be attributed to an increase of the interfacial viscosity $\eta_{\mathrm{s}}$ because of the presence of polymers at the interface. We provide estimates of $\eta_{\mathrm{s}}$ which are the same order of magnitude as the interfacial viscosities reported for polymers at other fluid-fluid interfaces.

We study the rupture of a liquid crystal, 5CB, in an aqueous solution of a short chain polymer (poly (vinyl alcohol): PVA, $\bar{M}_{\mathrm{w}}=104500 \mathrm{~g} \cdot \mathrm{mol}^{-1}$, Sigma Aldrich). This combination of liquids allows to obtain droplets of liquid crystal in water which are at the basis of switchable films [14] and can be used for the study of finite-size effects in the formation of defects in confined liquid crystals [15]. The polymer used here is known to be surface active at the liquid-crystal water interface, a property used to fix the orientation of the LC director near the surface $[14,15]$. Experiments are carried out in a microfluidic flow-focusing set-up described previously [8]. The orifice through which $5 \mathrm{CB}$ and the aqueous solutions are forced was $120 \mu \mathrm{m}$ wide and $150 \mu \mathrm{m}$ high. The flow rate of $5 \mathrm{CB}$ is kept constant at $20 \mu \mathrm{l} \cdot \mathrm{h}^{-1}$. The external aqueous solution is supplied at a flow rate $0.5 \mathrm{ml} \cdot \mathrm{h}^{-1}<Q<6 \mathrm{ml} \cdot \mathrm{h}^{-1}$.

When the outer phase contains only PVA, 5CB wets the walls of the channel and forms long filaments (fig. 1(a)). Some drops actually detach but stick to the walls of the 

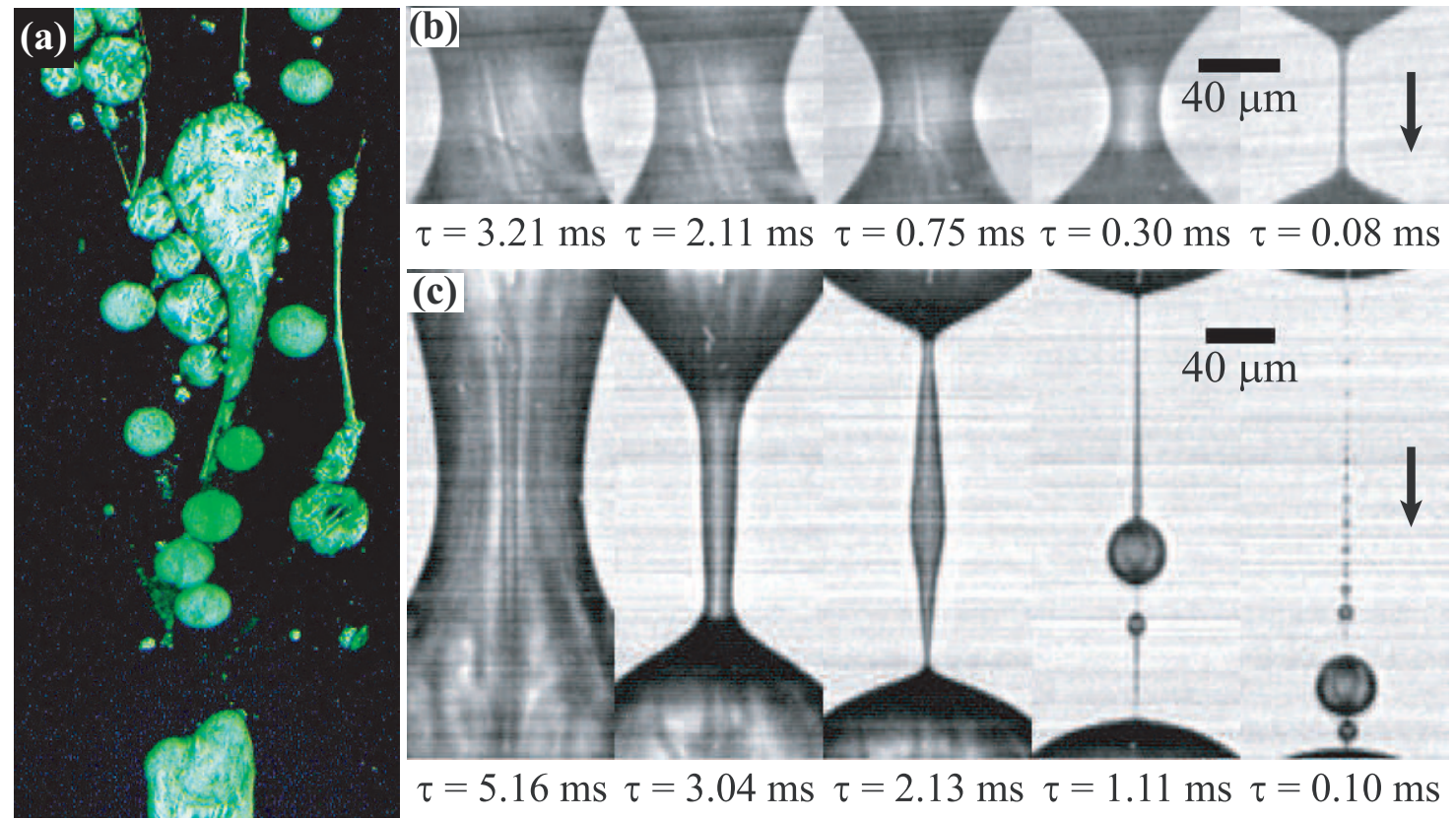

$\tau=5.16 \mathrm{~ms} \tau=3.04 \mathrm{~ms} \tau=2.13 \mathrm{~ms} \tau=1.11 \mathrm{~ms} \tau=0.10 \mathrm{~ms}$

Fig. 1: (Colour on-line) (a) Image of the liquid crystal 5CB dripping in a channel in the presence of PVA only, [PVA] $=1$ wt\%. (b) Thinning of a liquid-crystal neck in a pure SDS solution, $[\mathrm{SDS}]=0.1 \mathrm{cmc}$. (c) The same as (b) in a solution of SDS, $[\mathrm{SDS}]=0.1 \mathrm{cmc}$ with $1 \mathrm{wt} \%$ PVA. Times are indicated with respect to the break-up time, $t_{0}$. The black arrow indicates the direction of flow.

channel, which ends up being clogged. In order to avoid such effects we have added surfactant (sodium dodecyl sulfate: SDS, $M_{\mathrm{w}}=288.35 \mathrm{~g} \cdot \mathrm{mol}^{-1}$, Sigma Aldrich, $98 \%$ pure) to the aqueous solution of water and PVA. We will show later that PVA and SDS are competing to cover the 5CB-water interface with the PVA coverage increasing for low surfactant concentrations. Thus, to study the impact of PVA on the rupture process, we keep its concentration high and constant, $[\mathrm{PVA}]=1 \mathrm{wt} \%$, while we vary the concentration of SDS, $0.08 \mathrm{cmc}<[\mathrm{SDS}]<2 \mathrm{cmc}$ $(\mathrm{cmc}=8.2 \mathrm{mM})$. As we will see below, the effects of the polymer become manifest for small enough surfactant concentrations. The viscosity of the aqueous solutions $\eta_{\text {sol }}$, measured with a capillary viscometer, is $2 \mathrm{mPa} \cdot \mathrm{s}$ and does not change when varying [SDS]. The temperature is set at $27^{\circ} \mathrm{C}$, at which $5 \mathrm{CB}$ was in the nematic phase [16]. The viscosity of $5 \mathrm{CB} \eta_{5 \mathrm{CB}}$ depends on the angle between the direction of flow and the director of orientation $\vec{n}$ of the liquid crystal $[17,18]$. Here, $\vec{n}$ is set by the presence of SDS and PVA at the interface [14]. Rearrangement of the liquid-crystal molecules during pinch-off can also influence thinning dynamics [19]. Using polarizing microscopy observations, we find that $\vec{n}$ points in a direction perpendicular to the direction of the flow during all of the thinning process. Therefore, we assume that $\eta_{5 \mathrm{CB}} \simeq 80 \mathrm{mPa} \cdot \mathrm{s}[20,21]$ and remains constant. Both PVA and SDS are insoluble in 5CB. A fast camera mounted on a microscope records pinch-off events at frame rates between 2000 and 50000 frames per second, with a resolution as good as $0.31 \mu \mathrm{m}$ per pixel. The thinning dynamics of the neck are extracted by performing image analysis using a custom MatLab code.

Pinch-off in the presence of surfactants alone has been investigated mostly with numerical simulations [5-7]. A major finding of these studies is that surfactants may be depleted from the region around $h_{\min }$. This depletion leads to heterogeneities in the surfactant coverage of the interface and to the rise of capillary forces along the neck, which will influence rupture. In a previous work [8], we provided experimental evidence that surfactants cover non-uniformly the interface between two fluids during pinch-off and induce non-trivial thinning dynamics. At the location of fastest pinching, the interfacial tension $\gamma$, which is proportional to the thinning rate, can become greater than the interfacial tension at equilibrium, $\gamma_{\mathrm{eq}}$. This difference between $\gamma$ and $\gamma_{\text {eq }}$ demonstrates the occurrence of depletion. However, apart from a local increase of the surface tension, surfactants do not change the thinning dynamics drastically.

The effect of the presence of the short-chain polymer, PVA, in the system is readily seen by comparing the shapes of the liquid-crystal neck during pinch-off at a fixed but low SDS concentration [SDS] $=0.1 \mathrm{cmc}$, with and without PVA (fig. 1(b) and (c)). In the absence of polymer (fig. 1(b)), the neck thins at one location near the center of the drop during all of the thinning process. When the polymer is present, the neck becomes roughly cylindrical (image at $\tau=3.04 \mathrm{~ms}$ in fig. 1(c)), and then thins at two different locations, one close to the liquid-crystal reservoir and the other close to the main drop. As thinning 

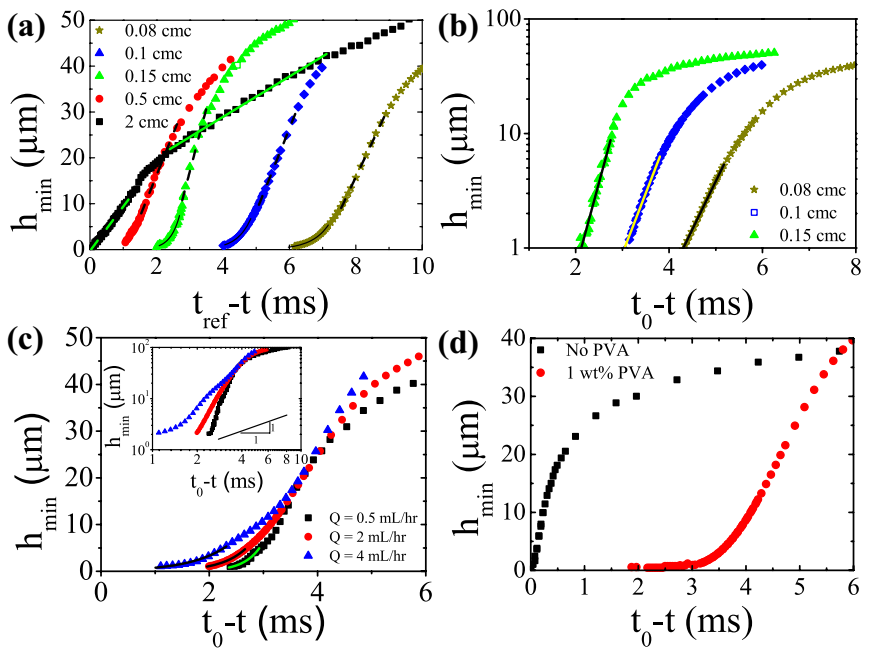

Fig. 2: (Colour on-line) (a) Comparison of the thinning dynamics $h_{\text {min }}$ for different concentrations of SDS in the presence of PVA, $[\mathrm{PVA}]=1 \mathrm{wt} \%$. For $[\mathrm{SDS}]=2 \mathrm{cmc}$, note that the dynamics can be represented by two linear parts with different slopes: a small slope at long times away from break-up and a higher slope near break-up. For the other concentrations, the continuous lines are exponential fits to the data, while the dashed lines are the linear parts of the thinning dynamics. $t_{\mathrm{ref}}$ is the time at break-up, $t_{0}$, to which we added $0.5 \mathrm{~ms}$ for $[\mathrm{SDS}]=0.5 \mathrm{cmc}$, $1 \mathrm{~ms}$ for $[\mathrm{SDS}]$ and $2 \mathrm{~ms}$ for $[\mathrm{SDS}]=0.08 \mathrm{cmc}$ to separate the different data sets. (b) Log-linear plot of the thinning dynamics. (c) Thinning dynamics for different flow rates of the external phase, $Q$. The thinning curves have been represented with respect to the break-up time $t_{0}$ for convenience. The inset shows log-log plots of the thinning dynamics. (d) Comparison of the thinning dynamics of $5 \mathrm{CB}$ in a solution containing $[\mathrm{SDS}]=0.1 \mathrm{cmc}$ with and without PVA.

proceeds, small droplets connected by threads grow on the neck and form a beads-on-a-string structure (image at $\tau=0.10 \mathrm{~ms}$ in fig. $1(\mathrm{c}))$. This effect can become drastic for $[\mathrm{SDS}]<0.1 \mathrm{cmc}$, as break-up slows down considerably and the length of the fluid neck can become comparable to the length of the channel.

A study of the thinning dynamics of the neck shows that the observation of the effects induced by the presence of PVA depends on $[\mathrm{SDS}]$. For $[\mathrm{SDS}] \geqslant 0.5 \mathrm{cmc}$, we see no beads-on-a-string structure. Moreover, the thinning dynamics are described by a series of linear regimes (fig. 2(a)) in a fashion very similar to what we had observed with external phases containing SDS only [8]. This multiple-regime process is due to the temporal variation of the interfacial tension $\gamma$ in the vicinity of the region around $h_{\text {min }}$ during pinch-off. From the thinning dynamics, $\gamma$ can be estimated using the two-fluid linear thinning law [22-24], $h_{\min }(t)=V\left(t_{0}-t\right)$, where $t_{0}$ is the time at which $h_{\min }$ becomes zero and $V$ is the thinning velocity. For an outer fluid with viscosity $\eta_{\text {out }}$ having an interfacial tension $\gamma$ with an inner fluid with viscosity $\eta_{\text {in }}, V$ is given by $V=\mathcal{H} \gamma / \eta_{\text {out }}$. The viscosity contrast $\eta_{\text {in }} / \eta_{\text {out }}=\eta_{5 \mathrm{CB}} / \eta_{\text {sol }} \simeq 40$ sets the value of $\mathcal{H}$, which is roughly $2 \cdot 10^{-3}$ in our case [24]. For $[\mathrm{SDS}]=2 \mathrm{cmc}$ (see fig. 2(a)), we find $V_{1}=8.2 \pm 2.3 \mathrm{~mm} \cdot \mathrm{s}^{-1}$ for the linear regime far from break-up and $V_{2}=20.9 \pm 0.5 \mathrm{~mm} \cdot \mathrm{s}^{-1}$ in the last instants before rupture. From this, we deduce $\gamma_{1}=4.1 \pm 1.1 \mathrm{mN} \cdot \mathrm{m}^{-1}$ and $\gamma_{2}=10.4 \pm 0.2 \mathrm{mN} \cdot \mathrm{m}^{-1}$, in good agreement with what we had found in our previous work in the absence of PVA [8]: the initial surface tension is close to its equilibrium value while near rupture the surface tension increases. The increase of the surface tension, responsible for the increase in the thinning rate, is due to the depletion in SDS of the interface in the last instants before rupture. For $[\mathrm{SDS}]=0.5 \mathrm{cmc}$, similar results are obtained with $\gamma_{1}=8.4 \pm 1.5 \mathrm{mN} \cdot \mathrm{m}^{-1}$ and $\gamma_{2}=$ $18.3 \pm 2.2 \mathrm{mN} \cdot \mathrm{m}^{-1}$. Since this concentration is below the cmc, $\gamma_{1}$ is larger than at $2 \mathrm{cmc}$ as expected. The surface tension before rupture again shows that depletion is at work.

The good comparison between the values of $\gamma$ obtained here and those we reported in the case of pure SDS [8] indicates that the surface properties seem to be governed by the presence of surfactant only for $[\mathrm{SDS}] \geqslant 0.5 \mathrm{cmc}$ : PVA has little to no influence on pinch-off.

The thinning dynamics of the neck change dramatically when the surfactant concentration is further reduced below $0.5 \mathrm{cmc}$ (fig. 2(a)). Far from the break-up time, the thinning dynamics remains linear vs. time but with a slope that decreases as [SDS] decreases. This observation means that the interfacial tension $\gamma$ (the thinning velocity is proportional to $\gamma$ ) is reduced when [SDS] decreases, which is opposite to what would be expected if only SDS was present. This strongly suggests that the coverage of the interface by the polymers increases as [SDS] is decreased.

Below $h_{\text {min }} \sim 10 \mu \mathrm{m}$, the thinning dynamics slow down and are best approximated by $h_{\min }(t)=h_{0} \exp (-\alpha t)$ (fig. 2(b)) instead of the expected linear regime. Attempts at approximating the thinning dynamics using a power law turned out to be unsuccessful giving exponents higher than 1 or no power law (inset of fig. 2(c)). The exponential decay rate $\alpha$ depends on the degree of polymer coverage and decreases from $3500 \mathrm{~s}^{-1}$ to $1900 \mathrm{~s}^{-1}$ as [SDS] is decreased from $0.15 \mathrm{cmc}$ to $0.08 \mathrm{cmc}$ at a fixed flow rate $Q=0.5 \mathrm{ml} \cdot \mathrm{h}^{-1}$. Increasing $Q$ from $0.5 \mathrm{ml} \cdot \mathrm{h}^{-1}$ to $4 \mathrm{ml} \cdot \mathrm{h}^{-1}$ at a fixed concentration $[\mathrm{SDS}]=0.1 \mathrm{cmc}$ also leads to a decrease in $\alpha$ from $3200 \mathrm{~s}^{-1}$ to $1800 \mathrm{~s}^{-1}$ (fig. 2(c)). Figure 2(d), which compares the thinning dynamics in two solutions containing $[\mathrm{SDS}]=0.1 \mathrm{cmc}$ with and without polymers (as in figs. 1(b) and (c)), shows clearly that the exponential thinning and therefore the slowing-down are observed only in the presence of PVA. Note that thinning occurs identically both close to the main droplet and close to the reservoir.

All of these results indicate that the observations reported in figs. 1 and 2 are related to the presence of PVA in the system and to its ability to cover the $5 \mathrm{CB}$-water interface. Note that our observations can be explained only by the influence of the polymers present at the interface. If the polymers in the bulk of the external 

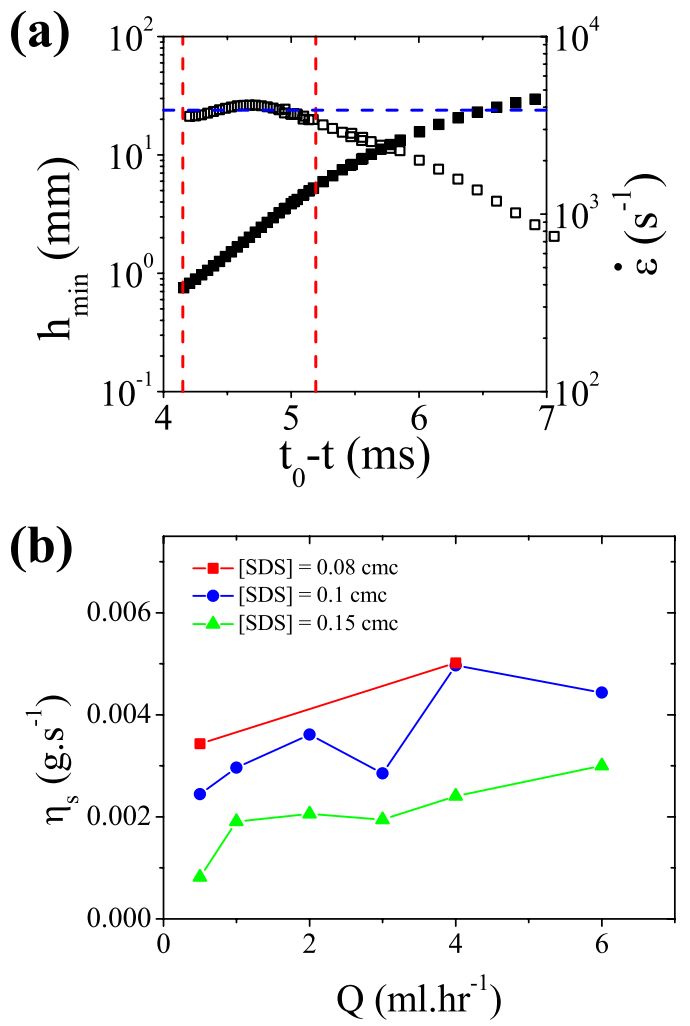

Fig. 3: (Colour on-line) (a) Thinning dynamics (closed squares) and elongation rate $\dot{\epsilon}$ (open squares) vs. time. (b) Interfacial viscosity $\eta_{\mathrm{s}}$ for different SDS concentrations in the presence of PVA vs. the flow rate of the external phase, $Q$.

phase affected pinch-off, we would observe different thinning dynamics for the rupture of $5 \mathrm{CB}$ in solutions containing $[\mathrm{SDS}] \geqslant 0.5 \mathrm{cmc}$ with and without PVA, which we do not. Also, the polymer is insoluble in the inner liquid $(5 \mathrm{CB})$ so effects due to polymers in the bulk of the rupturing fluid are ruled out.

To illustrate the validity of the exponential approximation to the thinning dynamics of the necks, we estimate the extensional rate, $\dot{\epsilon}=-\frac{2}{h_{\min }} \frac{\mathrm{d} h_{\min }(t)}{\mathrm{d} t}$. We extract $\dot{\epsilon}$ from spline fits to the thinning dynamics. The extensional rate $\dot{\epsilon}$ increases as the system gets closer to pinch-off and reaches a quasi-plateau value during the slowing-down (fig. 3(a)). A simple calculation indicates that $\dot{\epsilon}$ must be constant during an exponential regime and proportional to the decay rate $\alpha, \dot{\epsilon}=2 \alpha$. For the case shown in fig. 3, the calculated value of $\dot{\epsilon}$ is $4400 \mathrm{~s}^{-1}$, which compares very well with the plateau value we measure, $\dot{\epsilon}_{\mathrm{p}} \simeq 4500 \mathrm{~s}^{-1}$. The agreement between the two values of $\dot{\epsilon}$ suggests that the thinning dynamics can be correctly approximated by an exponential law.

Growth of beads-on-a-string structures, an exponential slowing-down of the thinning dynamics and the inhibition of break-up have been observed during the pinch-off of bulk polymeric solutions and attributed to the stretching of polymers in the neck region and to the importance of an elongational viscosity $[10-13]$. Besides the fact that the polymer is insoluble in the $\mathrm{LC}$, our results actually rule out an interpretation invoking an elongational viscosity as for bulk polymer solutions. Indeed, by balancing the capillary stress and the extensional stress during the exponential regime, we can estimate the elongational viscosity, $\eta_{\mathrm{e}}=$ $\gamma /\left(h_{\min } \dot{\epsilon}\right)$. For some of the runs analyzed in this way, the computed value of $\eta_{\mathrm{e}}$ comes out to be smaller than the elongational viscosity of $5 \mathrm{CB}$ estimated in our case, $\eta_{\mathrm{e}, 5 \mathrm{CB}}=3 \eta_{5 \mathrm{CB}}$. This result rules out this approach as the addition of the PVA layer should not decrease the extensional viscosity.

As we have mentioned earlier, the short-chain polymer used here, PVA, is insoluble in the rupturing liquid crystal, and the changes of the thinning dynamics occur only when the polymer coverage of the interface is important. Therefore, we propose another explanation to our observations based on a balance involving the interfacial tension $\gamma$ and the interfacial viscosity $\eta_{\mathrm{s}}$. In this framework, a time scale $\omega$ appears naturally, $\omega \sim \gamma / \eta_{\mathrm{s}}$, the units of $\eta_{\mathrm{s}}$ being $\mathrm{g} \cdot \mathrm{s}^{-1}$. Then, any continuous function $f(\omega t)$ may represent the thinning dynamics, the exponential function being a simple example. We identify $\omega$ to $\dot{\epsilon}$ and, by extracting the values of the interfacial tension from the linear regime preceding the slowing-down (from a measurement of the thinning velocity), we obtain estimates of $\eta_{\mathrm{s}}$ as a function of the flow rate of the external phase $Q$ for $[\mathrm{SDS}]=0.08$, 0.1 and $0.15 \mathrm{cmc}$ (fig. 3). These estimates are on the order of a few $10^{-3} \mathrm{~g} \cdot \mathrm{s}^{-1}$, which is the same order of magnitude as the interfacial viscosities reported in the literature for polymers at fluid-fluid interfaces $[25,26]$. Note that we are not aware of measurements of $\eta_{\mathrm{s}}$ for PVA at the 5CB-water interface. A decrease in [SDS] increases $\eta_{\mathrm{s}}$ threefold and is therefore related to the increase of polymer coverage of the surface as the concentration of surfactant decreases. The interfacial viscosity also increases mildly with $Q$.

As noted above, the onset of the exponential regime occurs only below about $10 \mu \mathrm{m}$. At larger neck radii, the dynamics is set by the balance between viscous and capillary stresses and the thinning dynamics remains linear as expected. The balance between the interfacial viscosity and capillarity that we propose for the small radii implies that the interfacial viscous stress becomes greater than the bulk viscous stress near rupture which can be summed up in the criterion $\eta_{\mathrm{s}} / \eta_{\text {in }} h_{\min } \gg 1[27,28]$. Here, this condition is true only if $h_{\min } \ll 50 \mu \mathrm{m}$ for $\eta_{\mathrm{s}}=4 \times 10^{-3} \mathrm{~g} \cdot \mathrm{s}^{-1}$. This criterion is well verified in our experiments, as the onset of the exponential regime occurs at about $h_{\min }=10 \mu \mathrm{m}$.

In conclusion, we show that the presence of surfaceactive polymers at the interface between two fluids is sufficient to affect pinch-off. The thinning dynamics slows down exponentially as break-up is approached. The final instants show the formation of beads-on-a-string structures. These observations are usually reported for bulk polymeric fluids led to rupture. We argue that such dynamics occurs when capillary pressure is balanced by 
a purely interfacial viscous stress. We extract the relevant surface viscosities from the experimental thinning curves and show that our estimates are in agreement with previous measurements. These results show that the use of polymers as surface-active agents can impair the production of dispersions either by affecting the polydispersity of the emulsion through the formation of beads on a string structures or by inhibiting the pinch-off singularity which leads to the detachment of droplets.

The authors would like to acknowledge H. A. STOnE for discussions and for making us aware of the role of the surface viscosity.

\section{REFERENCES}

[1] Eggers J., Rev. Mod. Phys., 69 (1997) 865.

[2] Eggers J. and Villermaux E., Rep. Prog. Phys., 71 (2008) 036601.

[3] XU J. H. et al., Langmuir, 22 (2006) 7943.

[4] Timmermans M.-L. E. and Lister J. R., J. Fluid Mech., 459 (2002) 289.

[5] Ambravaneswaran B. and Basaran O. A., Phys. Fluids, 11 (1999) 997; LiaO Y.-C., Franses E. I. and Basaran O. A., Phys. Fluids, 18 (2006) 022101; McGough P. T. and Basaran O. A., Phys. Rev. Lett., 96 (2006) 054502; Xu Q., LiaO Y.-C. and Basaran O. A., Phys. Rev. Lett., 98 (2007) 054503.

[6] Jin F., Gupta N. R. and Stebe K. J., Phys. Fluids, 18 (2006) 022103.

[7] Hameed M., Siegel M., Young Y.-N., Li J., Booty M. R. and Papageorgiou D. T., J. Fluid Mech., 594 (2008) 307; Young Y.-N., Booty M. R., Siegel M. and Li J., Phys. Fluids, 21 (2009) 072105.
[8] Roché M. et al., Phys. Rev. Lett., 103 (2009) 264501.

[9] Spigone E. et al., Langmuir, 25 (2009) 7457; Auguste D. T. et al., Langmuir, 24 (2008) 4056.

[10] Amarouchene Y. et al., Phys. Rev. Lett., 86 (2001) 3558.

[11] Oliveira M. S. N. and McKinley G. H., Phys. Fluids, 17 (2005) 071704.

[12] Sattler R., Wagner C. and Eggers J., Phys. Rev. Lett., 100 (2008) 164502.

[13] Arratia P. E., Gollub J. P. and Durian D. J., Phys. Rev. E, 77 (2008) 036309.

[14] Drzaic P. S., Liquid Crystal Dispersions (World Scientific Publishing Company) 1995.

[15] Rudhardt D. et al., Appl. Phys. Lett., 82 (2003) 2610; Hsu P., Poulin P. and Weitz D. A., J. Colloid Interface Sci., 200 (1998) 182.

[16] Rai P. K., Denn M. M. and Maldarelli C., Langmuir, 19 (2003) 7370.

[17] Skarp K., Lagerwall S. T. and Stebler B., Mol. Cryst. Liq. Cryst., 60 (1980) 215.

[18] Chmielewsiki A. G., Mol. Cryst. Liq. Cryst., 132 (1986) 339.

[19] Savage J. R. et al., Soft Matter, 6 (2010) 892.

[20] Negita K., J. Chem. Phys., 105 (1996) 7837.

[21] Wu M.-H. et al., J. Soc. Rheol. Jpn., 31 (2003) 91.

[22] Lister J. R. and Stone H. A., Phys. Fluids, 10 (1998) 2758.

[23] Cohen I. et al., Phys. Rev. Lett., 83 (1999) 1147.

[24] Cohen I. and Nagel S. R., Phys. Fluids, 13 (2001) 3533.

[25] Barentin C. et al., J. Fluid Mech., 397 (1999) 331; Barentin C. et al., Eur. Phys. J. E, 2 (2000) 153.

[26] Langevin D. and Monroy F., Curr. Opin. Colloid. Interface Sci., 15 (2010) 283.

[27] Danov K. et al., J. Colloid Interface Sci., 175 (1995) 36.

[28] Schwartz D. K., Knobler C. M. and Bruinsma R., Phys. Rev. Lett., 73 (1994) 2841. 